\title{
The Effect of Masseter Activity Patterns during Chewing on Suprahyoid Activity in Subsequent Chewing Cycles
}

\author{
Yozo Miyaoka $^{{ }^{*}}$, Ichiro Ashida ${ }^{1}$, Hajime Iwamori ${ }^{1}$, Yuko Tamaki ${ }^{2}$, \\ Shin-ya Kawakami ${ }^{1}$, Takako Yamazaki ${ }^{1}$, Naoko Ito ${ }^{1}$ \\ ${ }^{1}$ Department of Health and Nutrition, Niigata University of Health and Welfare, Niigata, Japan \\ ${ }^{2}$ Department of Health and Nutrition, University of Niigata Prefecture, Niigata, Japan \\ Email: *miyaoka@nuhw.ac.jp
}

Received December 26, 2013; revised January 5, 2014; accepted January 14, 2014

Copyright (c) 2014 Yozo Miyaoka et al. This is an open access article distributed under the Creative Commons Attribution License, which permits unrestricted use, distribution, and reproduction in any medium, provided the original work is properly cited. In accordance of the Creative Commons Attribution License all Copyrights @ 2014 are reserved for SCIRP and the owner of the intellectual property Yozo Miyaoka et al. All Copyright (C) 2014 are guarded by law and by SCIRP as a guardian.

\begin{abstract}
Few studies have evaluated the effects of activity patterns of the jaw closing muscles assessed by specific parameters on jaw opening in subsequent cycles during the chewing of food. The objective of this study was to quantitatively analyze the effect of the masseter (jaw closer) activity patterns on suprahyoid (jaw opener) activity during subsequent cycles. The assessments were performed while participants naturally chewed six test foods that differed in size dimensions and textural properties. Surface electromyograms of the masseter (on the habitual working side) and suprahyoid muscles were recorded in ten healthy young adults, each of whom randomly received one of the six test foods. The activity patterns were assessed using three parameters specifically developed for their quantification. Changes in suprahyoid activity during each of the subsequent chewing cycles were examined by three amplitudinal (minimum, maximum, and net values of the integrated suprahyoid electromyogram) parameters and one durational (active duration) parameter. The main finding was that two of the three activity pattern parameters had a statistically significant effect only on the three amplitudinal parameters in three of the six test foods. These results suggest that masseter activity patterns partially affect suprahyoid activity during subsequent chewing cycles and that the effect is food dependent. A possible neural mechanism responsible for this effect is presented.
\end{abstract}

\section{KEYWORDS}

Masseter; Activity Pattern; Suprahyoid; Textural Property; Chewing; Adult

\section{Introduction}

Cyclical jaw opening and closing form the basis of the rhythmic chewing movement noted in mammalian species. The lateral pterygoid and suprahyoid (SH; anterior belly of the digastric, geniohyoid and mylohyoid) muscles constitute the jaw opening muscles (or jaw openers), while the temporalis, masseter (Mass) and medial pterygoid muscles serve as the jaw closing muscles (or jaw closers). Chewing movements, which usually begin with jaw opening and are followed by jaw closing, have been noted among many mammalian species, including humans [1-3].

Two groups of nuclei, the paragigantocellular and gi${ }^{*}$ Corresponding author. gantocellular reticular nuclei, and the medial bulbar reticular formation, located in the region of the medulla oblongata, are believed to play essential roles in the central pattern generator that controls the basic chewing rhythm [1-3]. Neurons of either or both nucleus groups send action potentials, via the parvocellular reticular nucleus, to the jaw opening and closing motoneurons, innervating the muscles responsible for chewing movement [4].

Activity of the jaw opening muscles during fictive chewing affects the activity of the jaw closing muscles via action potentials from the sensory apparatus of the muscle spindles and periodontal pressoreceptors, which are located in the orofacial region of animals [5-8] including humans [9-11]. Opening of the jaw stretches the muscle spindles of the jaw closing muscles, and the 
stretch reflex then contracts the muscles; conversely, closing of the jaw muscles during chewing is unlikely to affect jaw opening muscle activity [12], partly due to the lack of muscle spindles in the jaw opening muscles. Previous studies have exclusively considered the influence of jaw closing muscle activity on amplitudinal and/or durational parameters of jaw opening muscle activity, either in experimental animals [5-8] or in humans [9-11]. Few studies have examined the influence of jaw closing muscle activity patterns using specific parameters for assessment as opposed to visual observation.

The present study was designed to examine the functional relationships between the jaw closing and opening muscles using the following steps: first, quantitatively assessing Mass activity patterns using the $\mathrm{T}_{\mathrm{P}}$ technique $[13,14]$; second, evaluating $\mathrm{SH}$ activity changes in amplitudes and active (or burst) durations during subsequent chewing cycles; and finally, examining the statistical and functional relationships between jaw closing and opening muscle activity patterns using a linear model (LM) analysis.

\section{Materials and Methods}

\subsection{Participants}

Ten healthy young adults (seven men and three women; 19 - 21 years old) participated in the present experiment after providing informed consent. The experiments were approved by the Ethics Committee of the Niigata University of Health and Welfare.

\subsection{Test Foods}

The shape and textural data of the six test foods used in this study have been detailed previously [15]: 1) cheese (processed); 2) gummy candy; 3) marshmallow; 4) prune (dried); 5) rice cracker; and 6) sponge cake. The food shapes (Table 1; see Table 1 in [15] for details) differed from one another because the test foods were commercial products; most were not processed further in an effort to standardize their shapes and because such processing might have changed their properties. Only the cheese was processed further by cutting the contents of one package into two homomorphic pieces. Their dimensions, namely, length, width and height, were determined based on how each food was positioned in the mouth before chewing. For example, height was defined as the distance between the molars gripping the food. Three textural properties (hardness, cohesiveness and adhesiveness) of the test foods were measured (Table 2 in [15]) using a Texture Profile Unit (TPU-2S, Yamaden Inc., Tokyo, Japan) attached to a cylinder-shaped plunger, with a diameter of $8 \mathrm{~mm}$ at a room temperature of approximately $25^{\circ} \mathrm{C}$. Each sample was compressed twice (speed $2.5 \mathrm{~mm} / \mathrm{s}$; clearance, $2.0 \mathrm{~mm}$ ) and was measured four to seven times repeatedly.

\subsection{Electrophysiological Recordings}

Two surface electromyograms (EMGs) were recorded according to our previous report [16]: i.e., one pair of adhesive electrodes (Ambu Inc., Blue Sensor, Baltorpbakken, Denmark) was attached to the skin just above the $\mathrm{SH}$ muscles, and the other pair was attached above the Mass muscle on the participant's habitual working side. The EMG signals were amplified, filtered (15 - $5000 \mathrm{~Hz}$ ), fully rectified and integrated ( $\tau=0.06 \mathrm{~s}$ ) using the Power Lab system (AD Instruments Pty Ltd., Power Lab/8 sp, Bella Vista, Australia). An accelerometer (Takei Scientific Instruments Co. Ltd., Tokyo, Japan) was attached to the skin of the chin to record the trajectory of jaw movement and to determine the jaw opening and closing phases of the chewing cycles. In addition to the EMG and accelerometer recordings, two video cameras (Logicool ${ }^{\circledR}$,

Table 1. Shape and textural data of the six foods analyzed in this study.

\begin{tabular}{|c|c|c|c|c|c|c|}
\hline \multirow{2}{*}{ Foods tested } & \multicolumn{3}{|c|}{ Shape dimensions } & \multicolumn{3}{|c|}{ Textural properties } \\
\hline & Length & Width & Height & Hardness & Cohesiveness & Adhesiveness \\
\hline Cheese & $28.6 \pm 0.15$ & $13.6 \pm 0.13$ & $13.2 \pm 0.04$ & $144 \pm 14.5$ & $0.308 \pm 0.054$ & $2.71 \pm 1.100$ \\
\hline Gummy candy & $15.0 \pm 0.24$ & $23.6 \pm 0.23$ & $9.50 \pm 0.33$ & $705 \pm 31.1$ & $0.721 \pm 0.039$ & $3.38 \pm 0.741$ \\
\hline Marshmallow & $23.0 \pm 0.39$ & $23.4 \pm 0.76$ & $23.0 \pm 0.71$ & $311 \pm 9.86$ & $0.689 \pm 0.015$ & $4.58 \pm 0.242$ \\
\hline Prune & $23.2 \pm 1.67$ & $30.9 \pm 0.69$ & $15.6 \pm 0.60$ & $349 \pm 19.5$ & $0.390 \pm 0.060$ & $1.36 \pm 0.207$ \\
\hline Rice cracker & $29.7 \pm 0.29$ & $30.4 \pm 0.05$ & $8.30 \pm 0.22$ & $672 \pm 58.1$ & $0.080 \pm 0.047$ & $2.98 \pm 0.735$ \\
\hline Sponge cake & $30.8 \pm 0.81$ & $30.4 \pm 0.75$ & $28.1 \pm 0.45$ & $611 \pm 56.2$ & $0.298 \pm 0.019$ & $2.23 \pm 0.259$ \\
\hline
\end{tabular}

Values indicate means \pm S.E.M. Units are millimeter for "Shape dimensions”, kPa for "Hardness” and kJ/m for “Adhesiveness”, respectively. No unit is for “Cohesiveness”. 
Qcam Communicate STX, Tokyo, Japan) were used to monitor the movement of the body, neck and head of each participant from the frontal and lateral sides.

\subsection{Procedures}

Each participant was seated on a chair in an electrically shielded room at ambient temperature. The participant was provided with instructions regarding the chewing tasks from a computer display placed on a desk in front of him or her. One of the six test foods was delivered randomly to the participant, and he or she was asked to chew it using a natural rhythm. The participant was not restricted with regard to the chewing side, although the electrodes for the Mass EMG were attached to the habitual chewing side. The participant was also asked to swallow when he or she felt that the food had been chewed fully. The participant was allowed to drink room temperature green tea (Ohi-ocha, Ito-en, Tokyo, Japan) after swallowing the test food. The trials were separated by one-minute intervals, and each experimental session with a particular participant consisted of seven to 12 trials. Each participant ingested all of the six test foods at least once. In total, each of the ten participants underwent 77 trials: ten trials with cheese; 12 trials each with gummy candy and prune; 14 trials each with marshmallow and sponge cake; and 15 trials with rice cracker.

\subsection{Data Analysis}

In each chewing sequence, the initial five full cycles after chewing commenced were analyzed. The first SH EMG of each chewing sequence was not counted because the major purpose of this study was to examine the effects of Mass activity patterns on SH parameters during subsequent chewing cycles. The beginning and end of the Mass EMG were defined as the appearance and disappearance of the bursts and as the corresponding jaw movement trajectories. One chewing cycle was defined as the interval between the beginning of a Mass EMG burst and that of the next. In this study, the duration of the SH EMG was calculated by subtracting the duration of the Mass EMG from the duration of the corresponding chewing cycle, as the beginning and end of the $\mathrm{SH}$ EMGs usually overlapped with the end and beginning of the Mass EMG. The calculation was especially useful when the appearance and disappearance of the SH EMG bursts were unclear and when the durations were identical to those of the SH EMG bursts if the appearance and disappearance were clear, as seen in Figure 1. The following four parameters were measured from the raw and integrated SH (inteSH) EMG data: 1) the "minimum" inteSH amplitude ("a" in Figure 1); 2) the "maximum" inteSH amplitude ("b”); 3) the "net" ("maximum" "minimum") inteSH amplitude; and 4) the active dura-

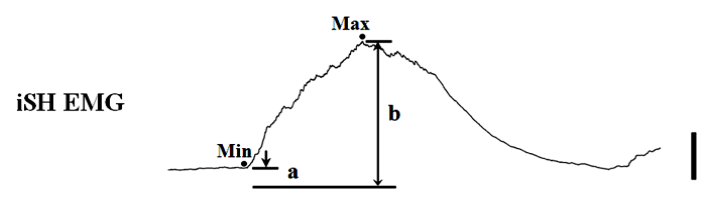

SH EMG

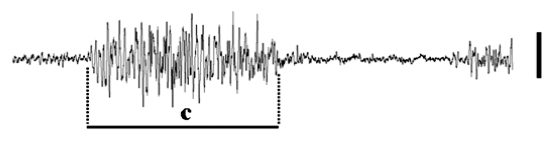

Figure 1. Data analysis. SH EMG and iSH EMG, raw and integrated electromyograms of the suprahyoid muscles, respectively. Vertical bars on the right indicate $3 \mathrm{mV} \cdot \mathrm{s}$ (for iSH EMG) and $0.2 \mathrm{mV}$ (for SH EMG and Mass EMG); a horizontal bar indicates $0.5 \mathrm{~s}$. A longer horizontal line below the iSH EMG indicates the value at baseline (i.e., 0 $\mathrm{mV} \cdot \mathrm{s})$, and the two shorter lines on iSH EMG indicate the minimum (Min, “a”) and maximum (Max, “b”) iSH EMG values, respectively. The active duration of the SH EMG ("c") was measured as the interval from the beginning to the end of the SH EMG. See the text for details.

tion ("c"). The "minimum" and "maximum" inteSH amplitudes were defined as the distances between the values at baseline (i.e., $0 \mathrm{mV} \cdot \mathrm{s}$ ) and the values at the start of the elevation (indicated by "Min" in Figure 1) and those at the peak (indicated by "Max") of the inteSH EMG, respectively. A chewing cycle was defined as the time interval between the start of the Mass EMG and the next one.

Three $T_{P}$ values $\left(T_{25}, T_{50}\right.$ and $\left.T_{75}\right)$ were calculated using the $T_{P}$ technique $[13,14]$ to quantify the activity patterns of the EMG; the technique was applied to the Mass EMG during the initial five cycles in each chewing sequence by taking the importance of the initial chewing cycles in motor control of jaw movement into consideration $[17,18]$. The outline of the $T_{P}$ technique is as follows: an EMG is cumulated from the beginning to the end of the burst; the cumulated EMG is divided into four equal sections; the duration from the beginning to the end of the burst is standardized as 1.000; the four sectioned values are serially projected onto the standardized time scale; and three $T_{P}$ values are designated as the projected values. According to the definition of the $T_{P}$ technique, $\mathrm{T}_{0}$ and $\mathrm{T}_{100}$ are always equal to 0.000 and 1.000 , respectively, and each $T_{P}$ value designates a relative location of the EMG on a standardized time scale. Consequently, the $\mathrm{T}_{\mathrm{P}}$ technique enables direct comparison of one activity pattern with another with different amplitudes and durations (see [13] for details).

\subsection{Statistical Analysis}

The LM analysis was applied to the EMG data obtained from each of the test foods. In this analysis, "three $T_{P}$ values" and "chewing cycles" were used as factors (ob- 
jective parameters). Each of the three $T_{P}$ values was used as a covariate in the LM analysis. An average chewing cycle was calculated by dividing the time for the five chewing cycles by five (i.e., the number of cycles). The three amplitudinal $\mathrm{SH}$ parameters and a durational $\mathrm{SH}$ parameter were used as explanatory parameters. Thus, a total of 24 (six test foods $\mathrm{x}$ four explanatory parameters) combinations were considered for ANOVA. The data for the LM analysis were initially corrected to account for the average number of "participants" because this factor was the only one expected to have a general effect on the results.

Because Bartlett's test detected some heterogeneity of variances in the data, two non-parametric tests, namely, the Kruskal-Wallis and Steel-Dwass tests, were used. First, the Kruskal-Wallis test was used to determine whether the six foods differed significantly in terms of the four parameters (i.e., " $\mathrm{T}_{25}$ values, $\mathrm{T}_{50}$ values, $\mathrm{T}_{75}$ values", and "chewing cycles"). Second, if the KruskalWallis test found a significant difference, the SteelDwass test was used to determine whether particular pairs of foods exhibited specific differences. Differences were considered to be statistically significant if $P<0.05$.

\section{Results}

Figure 2 shows sample data of the two EMGs collected from the beginning to the 5th cycle during the chewing of a prune in a male participant. The sample data suggest that the initial part of these Mass bursts diminished relatively from the 1 st to the 5 th chewing cycle. The $\mathrm{T}_{50}$ values calculated reflect that suggestion: i.e., 0.482 for the 1st cycle; 0.586 for the 3rd cycle; and 0.604 for the 5 th cycle. In accordance with the relative diminution of the $\mathrm{T}_{50}$ values, the bottom of the inte $\mathrm{SH}$ rose, and the minimum inte $\mathrm{SH}$ amplitude ( $\mathrm{a}_{1}$ to $\mathrm{a}_{5}$ in Figure 2 ) gradually increased.

The LM analysis revealed that four $T_{P}$ values (three $\mathrm{T}_{50}$ and one $\mathrm{T}_{75}$ ) had significant effects on the three amplitudinal parameters in three of the six test foods (Table 2): i.e., "minimum inteSH amplitude" during the chewing of a prune $\left(\mathrm{T}_{50}\right.$ and $\mathrm{T}_{75}$ values, $\left.P \mathrm{~s}<0.01\right)$; "maximum" inteSH amplitude with sponge cake $\left(\mathrm{T}_{50}, P<0.05\right)$, and "net inte $\mathrm{SH}$ amplitude" with cheese $\left(\mathrm{T}_{50}, P<0.05\right)$. The LM analysis also revealed that the "chewing cycles" factor was significant in four of the six test foods (Table 2): i.e., "minimum" inteSH amplitude during the chewing of rice cracker $(P<0.01)$, cheese, marshmallow, and prune $(P<0.05)$; "maximum" and "net" inteSH amplitudes with cheese $(P s<0.01)$, and "SH duration" with cheese $(P<0.05)$.

\section{Discussion}

The results indicated that Mass activity patterns affected
Table 2. Statistical significances detected in the individual test foods used in this study.

\begin{tabular}{|c|c|c|c|c|c|c|}
\hline Parameters & Cheese & $\begin{array}{l}\text { Gummy } \\
\text { candy }\end{array}$ & Marshmallow & Prune & $\begin{array}{c}\text { Rice } \\
\text { Cracker }\end{array}$ & $\begin{array}{l}\text { Sponge } \\
\text { Cake }\end{array}$ \\
\hline $\begin{array}{c}\text { Min SH } \\
\text { ampli }\end{array}$ & $\mathrm{C}^{*}$ & & $C^{*}$ & $\begin{array}{c}\mathrm{T}_{50}^{* *}, \\
\mathrm{~T}_{75}^{* *}, \mathrm{C}^{*}\end{array}$ & $C^{* *}$ & \\
\hline $\begin{array}{c}\text { Max SH } \\
\text { ampli }\end{array}$ & $C^{* *}$ & & & & & $\mathrm{~T}_{50}{ }^{*}$ \\
\hline $\begin{array}{l}\text { Net SH } \\
\text { ampli }\end{array}$ & $\mathrm{T}_{50}{ }^{*}, \mathrm{C}^{* *}$ & & & & & \\
\hline SH duration & $\mathrm{C}^{*}$ & & & & & \\
\hline
\end{tabular}

Min, minimum; Max, maximum; SH, suprahyoid; ampli, amplitude of the integrated SH EMG; $\mathrm{T}_{50}, \mathrm{~T}_{50}$ value; $\mathrm{T}_{75}, \mathrm{~T}_{75}$ value; $\mathrm{C}$, chewing cycle. ${ }^{* *} \mathrm{P}<$ $0.01,{ }^{*} P<0.05$

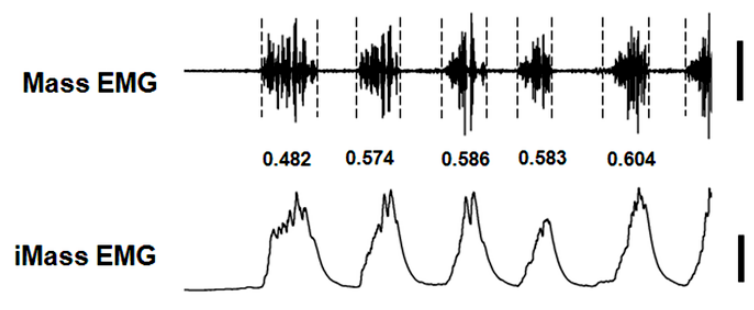

SH EMG

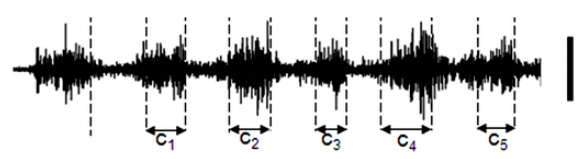

iSH EMG

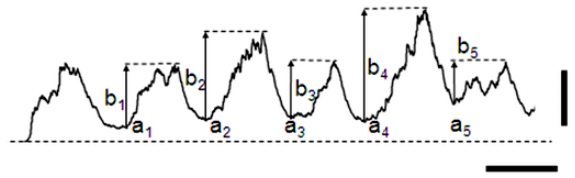

Figure 2. Sample data of masseter and suprahyoid muscle activities during the chewing of a prune. Mass EMG and iMass EMG, raw and integrated electromyograms of the masseter muscle, respectively; SH EMG and iSH EMG, raw and integrated electromyograms of the suprahyoid muscles, respectively. Numbers from 0.482 to 0.604 between the Mass and iMass EMGs indicate the $T_{50}$ values calculated by the $T_{P}$ technique. Vertical dotted lines indicate the beginning and end of the Mass EMG. See Figure 1 for " $a_{i}$ ", " $b_{i}$ " and " $c_{i}$ " ( $i=1$ to 5$)$ in SH and iSH EMGs. Note that the first SH EMG was not counted in this study. See the text for details. Vertical bars on the right indicate $1.0 \mathrm{mV}$ and 5 $\mathrm{mV} \cdot \mathrm{s}$ (for Mass and iMass EMGs) and $0.4 \mathrm{mV}$ and $2 \mathrm{mV} \cdot \mathrm{s}$ (for SH and iSH EMGs), respectively; a horizontal bar indicates $0.5 \mathrm{~s}$.

amplitudinal, but not durational, SH parameters during subsequent chewing cycles in three (cheese, prune, and sponge cake) of the six test foods (Table 2). There is only limited knowledge regarding activity pattern changes in the jaw closing and opening muscles, although qualitative changes have been described using visual observations $[5,6]$ and not specific, appropriate 
parameters for assessing activity patterns. Consequently, it is challenging to compare our results to those of other investigators. We developed $T_{P}$ values that were specific to the assessment $[13,14,18]$ and adopted the parameters $\left(T_{25}, T_{50}\right.$ and $T_{75}$ values) for the analysis in this study. As a result, we found only four significant effects of masseter $T_{P}$ values on the amplitudinal parameters of the $\mathrm{SH}$ muscles (Table 2). This finding suggests that masseter activity patterns can affect $\mathrm{SH}$ muscle amplitude during subsequent chewing cycles, but the effect is limited. Our results also suggest that the effect might be food dependent (Table 2).

A previous study in awake rabbits [8] recorded 12 trigeminal muscle activities during chewing, and the authors extracted three principal components related to the amplitude from the recorded data: the jaw closers, jaw openers and posterior deep masseter. The authors suggested that there was independent control over the jaw opening and closing muscles, and also between the amplitude and timing of the masticatory muscles during chewing. In healthy humans, a series of studies have been conducted that have simulated chewing experiments [9-11], in which the participants unexpectedly received changes in the simulated force used to mimic food resistance during natural jaw opening and jaw closing; the authors observed additional muscle activity in the jaw closers but not in the jaw openers. The findings from these studies [9-11] appear inconsistent with our results, which may in part be due to the largely experimental situations between the studies, i.e., the previous studies analyzed the influences of force changes on jaw opener activity concomitant with jaw closer, and not in subsequent cycles; these studies only stated that the additional muscle activity evoked no coactivation of the jaw openers.

As shown in Table 2, we also found that the "chewing cycles" factor had effects on all of the four explanatory parameters in four (cheese, marshmallow, prune and rice cracker) of the six test foods. The present study analyzed the initial five cycles of each chewing sequence; thus, we do not know whether the effects were sustained throughout the chewing sequence. In addition, the small samples of both significant and non-significant test foods made it difficult to infer the reason for the difference between the two groups of test foods, at least based on food parameters (Table 1). A previous study, using four visco-elastic model foods with different hardness, analyzed masticatory muscle parameters collected during chewing of the model foods and demonstrated chewing cycle-dependent changes in the muscle parameters. This finding highlights the importance of the initial five chewing cycles when evaluating the effects of food hardness on physiological parameters [18]. Consistent with our results, these studies suggest that food hardness can affect not only jaw closing muscle activity during the initial five chewing cycles, but also jaw opening muscle activity, which is followed by jaw closing muscle activity.

\section{Conclusion}

$T_{P}$ values quantitatively assessed masseter activity patterns during the chewing of six test foods by ten healthy young adults. The masseter activity patterns partially affected the suprahyoid amplitude during subsequent chewing cycles in only two of the test foods. The chewing cycles also affected suprahyoid activity during subsequent chewing cycles.

\section{Acknowledgements}

This study was supported in part by Grants-in-Aid for Scientific Research from the Ministry of Education, Science and Culture of Japan (No. 195000667 and No. 22500740 to YM, No. 23700889 to TY and No. 2050072 to IA).

\section{REFERENCES}

[1] Y. Nakamura and N. Katakura, "Generation of Masticatory Rhythm in the Brainstem," Neuroscience Research, Vol. 23, No. 1, 1995, pp. 1-19.

[2] S. Nozaki, A. Iriki and Y. Nakamura, "Role of Corticobulbar Projection Neurons in Cortically Induced Rhythmical Masticatory Jaw-Opening Movement in the Guinea Pig,” Journal of Neurophysiology, Vol. 55, No. 4, 1986, pp. 826-845.

[3] S. Nozaki, A. Iriki and Y. Nakamura, "Localization of Central Rhythm Generator Involved in Cortically Induced Rhythmical Masticatory Jaw-Opening Movement in the Guinea Pig,” Journal of Neurophysiology, Vol. 55, No. 4, 1986, pp. 806-825.

[4] Y. Yamada, K. Yamamura and M. Inoue, "Coordination of Cranial Motoneurons during Mastication,” Respiratory Physiology \& Neurobiology, Vol. 147, No. 2-3, 2005, pp. 177-189. http://dx.doi.org/10.1016/j.resp.2005.02.017

[5] T. Inoue, T. Kato, Y. Masuda, T. Nakamura, Y. Kawamura and T. Morimoto, "Modifications of Masticatory Behavior after Trigeminal Deafferentation in the Rabbit," Experimental Brain Research, Vol. 74, No. 3, 1989, pp. 579-591. http://dx.doi.org/10.1007/BF00247360

[6] O. Hidaka, T. Morimoto, Y. Masuda, T. Kato, R. Matsuo, T. Inoue, M. Kobayashi and K. Takada, "Regulation of Masticatory Force during Cortically Induced Rhythmic Jaw Movements in the Anesthetized Rabbit," Journal of Neurophysiology, Vol. 77, No. 6, 1997, pp. 3168-3179.

[7] Z. J. Liu, K. Ikeda, S. Harada, Y. Kasahara and G. Ito, "Functional Properties of Jaw and Tongue Muscles in Rats Fed a Liquid Diet after Being Weaned," Journal of Dental Research, Vol. 77, No. 2, 1998, pp. 366-376. http://dx.doi.org/10.1177/00220345980770020501

[8] W. A. Weijs, T. Sugimura and L. J. van Ruijven, "Motor Coordination in a Multi-Muscle System as Revealed by 
Principal Components Analysis of Electromyographic Variation," Experimental Brain Research, Vol. 127, No. 3, 1999, pp. 233-243. http://dx.doi.org/10.1007/s002210050793

[9] F. A. Ottenhoff, A. van der Bilt, H. W. van der Glas and F. Bosman, "Peripherally Induced and Anticipating Elevator Muscle Activity during Simulated Chewing in Humans,” Journal of Neurophysiology, Vol. 67, No. 1, 1992, pp. 75-83.

[10] F. A. Ottenhoff, A. van der Bilt, H. W. van der Glas and F. Bosman, "Control of Human Jaw Elevator Muscle Activity during Simulated Chewing with Varying Bolus Size,” Experimental Brain Research, Vol. 96, No. 3, 1993, pp. 501-512. http://dx.doi.org/10.1007/BF00234118

[11] A. van der Bilt, F. G. Weijnen, F. A. Ottenhoff, H. W. van der Glas and F. Bosman, "The Role of Sensory Information in the Control of Rhythmic Open-Close Movements in Humans," Journal of Dental Research, Vol. 74, No. 10, 1995, pp. 1658-1664. http://dx.doi.org/10.1177/00220345950740100601

[12] J. D. van Willigen, P. J. Juch, C. M. Ballintijn and M. L. Broekhuijsen, “A Hierarchy of Neural Control of Mastication in the Rat,” Neuroscience, Vol. 19, No. 2, 1986, pp. 447-455. http://dx.doi.org/10.1016/0306-4522(86)90273-3

[13] I. Ashida, S. Kawakami and Y. Miyaoka, "A New Method of Simulating Surface Electromyograms Using Probability Density Functions," Computers in Biology and Medicine, Vol. 38, No. 7, 2008, pp. 837-844. http://dx.doi.org/10.1016/j.compbiomed.2008.05.001

[14] Y. Miyaoka, I. Ashida, D. Inagaki and S. Kawakami, "Differentiation of Activity Patterns in the Suprahyoid Muscles during Swallowing of Foods with Five Taste Qualities,” Journal of Sensory Studies, Vol. 20, No. 6, 2005, pp. 473-483.

http://dx.doi.org/10.1111/j.1745-459X.2005.00041.X

[15] Y. Miyaoka, I. Ashida, Y. Tamaki, S. Y. Kawakami, H. Iwamori, T. Yamazaki and N. Ito, "Sequential Analysis of Masseter Activity Patterns during Chewing in Healthy Males,” Journal of Medical Engineering \& Technology, Vol. 37, No. 2, 2013, pp. 91-95. http://dx.doi.org/10.3109/03091902.2012.747007

[16] I. Ashida, H. Iwamori, S. Kawakami, Y. Miyaoka and A. Murayama, "Analysis of Physiological Parameters of Masseter Muscle Activity during Chewing of Agars in Healthy Young Males,” Journal of Texture Studies, Vol. 38, No. 1, 2007, pp. 87-99. http://dx.doi.org/10.1111/j.1745-4603.2007.00087.x

[17] C. Lassauzay, M. A. Peyron, E. Albuisson, E. Dransfield and A. Woda, "Variability of the Masticatory Process during Chewing of Elastic Model Foods,” European Journal of Oral Sciences, Vol. 108, No. 6, 2000, pp. 484-492. http://dx.doi.org/10.1034/j.1600-0722.2000.00866.x

[18] M. A. Peyron, C. Lassauzay and A. Woda, "Effects of Increased Hardness on Jaw Movement and Muscle Activity during Chewing of Visco-Elastic Model Foods,” Experimental Brain Research, Vol. 142, No. 1, 2002, pp. 41-51. http://dx.doi.org/10.1007/s00221-001-0916-5 